\title{
Functional Role of a Conserved Sequence Motif in the Oxygen-dependent Degradation Domain of Hypoxia-inducible Factor $1 \alpha$ in the Recognition of p53
}

\section{Seung-Wook Chi*}

Translational Research Center, Korea Research Institute of Bioscience and Biotechnology, Daejeon 305-806, Korea

\begin{abstract}
Hypoxia-inducible factor $1 \alpha$ (HIF1 $\alpha)$ is a transcription factor that plays a key role in the adaptation of cells to low oxygen stress and oxygen homeostasis. The oxygen-dependent degradation (ODD) domain of HIF1 $\alpha$ is responsible for the negative regulation of $\mathrm{HIF} 1 \alpha$ in normoxia. The interactions of the HIF1 $\alpha$ ODD domain with partner proteins such as von Hippel-Lindau tumor suppressor (pVHL) and p53 are mediated by two sequence motifs, the $\mathrm{N}$ - and C-terminal ODD (NODD and CODD). Multiple sequence alignment with HIF1 $\alpha$ homologs from human, monkey, pig, rat, mouse, chicken, frog, and zebrafish has demonstrated that the NODD and CODD motifs have noticeably high conservation of the primary sequence across different species and isoforms. In this study, we carried out molecular dynamics simulation of the structure of the HIF1 $\alpha$ CODD motif in complex with the p53 DNA-binding domain (DBD). The structure reveals specific functional roles of highly conserved residues in the CODD sequence motif of HIF1 $\alpha$ for the recognition of p53.
\end{abstract}

Keywords: hypoxia-inducible factor $1 \alpha$, molecular dynamics simulation, oxygen-dependent degradation domain, p53, sequence motif

\section{Introduction}

Hypoxia-inducible factor $1 \alpha$ (HIF1 $\alpha)$ is a master regulator in oxygen homeostasis and in the adaptation of cells to low oxygen stress (Bruick and McKnight, 2002; Lee et al., 2004; Semenza, 2001). HIF1 $\alpha$ is a ubiquitous, basic helix-loop-helix/PAS (Per, Arnt, and Sim protein) transcription factor that activates the expression of genes that are involved in angiogenesis, erythropoiesis, energy metabolism, apoptosis, and proliferation in

${ }^{*}$ Corresponding author: E-mail swchi@kribb.re.kr Tel +82-42-860-4277, Fax +82-42-860-4593 Accepted 13 May 2008 response to hypoxia (Semenza, 1999; Semenza, 2003). While it is stable in hypoxia, HIF1 $\alpha$, in normoxia, undergoes ubiquitination by von Hippel-Lindau tumor suppressor ( $\mathrm{pVHL}$ ) and subsequent proteasomal degradation. This sensing of change in oxygen levels is mediated by the oxygen-dependent degradation (ODD) domain of HIF1 $\alpha$ (Fig. 1). Binding of pVHL to the HIF1 $\alpha$ ODD domain is dependent on the hydroxylation of two prolyl residues, Pro ${ }^{402}$ and $\mathrm{Pro}^{564}$, in the ODD domain (Ivan et al., 2001; Jaakkola et al., 2001). On the other hand, the HIF1 $\alpha$ ODD domain exerts its transcriptional activation function through the interaction of its C-terminal part (residues 531 575, N-TAD) with basal transcription machinery (Jiang et al., 1996). Recently, the interactions of the HIF1 $\alpha$ ODD domain with multiple partner proteins, such as ARD1 (Jeong et al., 2002), prolyl hydroxylase (PHD) (Schofield and Ratcliffe, 2004), and p53 (Fels and Koumenis, 2005; Sanchez-Puig et al., 2005), also have been reported.

However, the molecular basis for the multiple binding specificity of the HIF1 $\alpha$ ODD domain has not been understood yet. The detailed characterization of the correlation between the binding sequence motifs in the ODD domain and its binding to multiple target proteins is necessary for understanding the versatile function of the HIF1 $\alpha$ ODD domain. Two functionally independent sequence motifs, the $\mathrm{N}$-terminal and C-terminal ODD (NODD and CODD), in the HIF1 $\alpha$ ODD domain were shown to bind to the DNA-binding domain (DBD) of p53 (Hansson et al., 2002). The crystal structure of the CODD motif in complex with pVHL was determined to

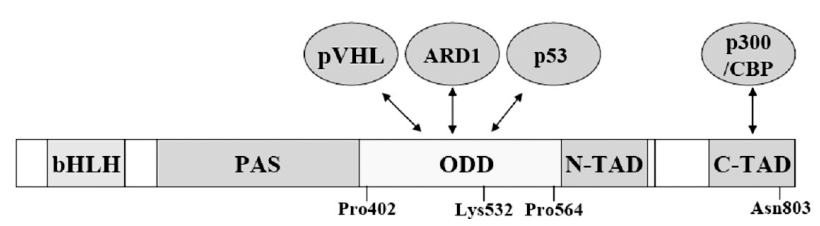

Fig. 1. Domain structure of HIF1 $\alpha$. Functional domains of HIF1 $\alpha$, their partner proteins, and protein modification sites are represented. "bHLH" and "PAS" indicate the basic helix-loop-helix and Per-Arnt-Sim, respectively. "N-TAD" and "C-TAD" represent the $\mathrm{N}$-terminal and $\mathrm{C}$-terminal transcriptional activation domain, respectively. $\mathrm{Pro}^{402}$, $\mathrm{Pro}^{564}$, and $\mathrm{Asn}^{803}$ (Lando et al., 2002) are hydroxylation sites, and Lys $^{532}$ is an acetylation site (Jeong et al., 2002). 
explain the structural basis for the recognition of hydroxyproline in the CODD motif by pVHL (Hon et al., 2002; Min et al., 2002). In this study, we performed molecular dynamics simulation of the structure of the complex between the CODD motif and the p53 DBD and compared it with the structure of the complex between the CODD motif and $\mathrm{pVHL}$. The results demonstrate specific functional roles of highly conserved amino acids in the HIF1 $\alpha$ ODD motif for the recognition of $\mathrm{p} 53$.

\section{Methods}

To build a structural model of a HIF1 $\alpha$ CODD peptide (residues $560 \sim 577$ ) in complex with the p53 DBD, we performed molecular dynamics simulation by using the molecular modeling package Insightll (Accelrys). The initial structure of the HIF1 $\alpha$ CODD peptide was obtained from the structure of the hydroxylated peptide bound to pVHL (PDB code 1LQB) (Hon et al., 2002). Based on the contact points from previous chemical shift perturbation data (Hansson et al., 2002), the CODD peptide was manually docked in an extended structure on the binding groove of the crystal structure of DNA-free p53 DBD (PDB code 2OCJ) (Wang et al., 2007). To get rid of steric clashes, conjugate gradient minimization of the modeled structure was performed by the program DISCOVER until $0.01 \mathrm{kcal} / \mathrm{mol} / \AA$ was reached. This was followed by 500 -ps molecular dynamics simulation at $300 \mathrm{~K}$, in which the backbones of the molecule were restrained. During the molecular dynamics simulation,

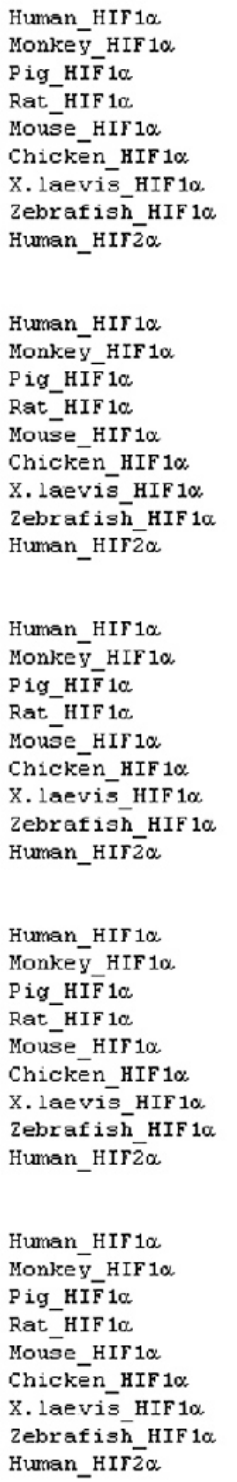

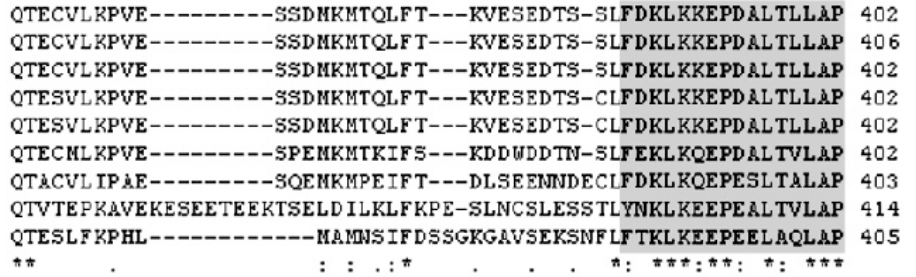

AAGDTI ISLDFGSNDTE TDDQQLE EVPL YNDVHLPSPNEKL QNINLAISPLPTAE TP KPL 452

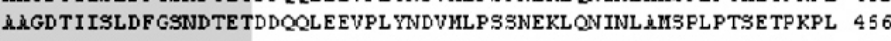
A $\&$ GDTI ISLDF GSNDTE TD DQQLEEVPL YNDVHLPSSNEKHONINLAMSPLPASE TP KPL 452 AAGDTI ISLDFGSDDTE TEDQQLE DVPL YNDVHF PSSNEKLN-INLAMSPLPASE TPKPL 451

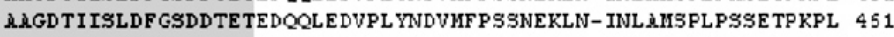
AMGDTI ISLDFSSN--ESDEQQCDEVPL YNDVHLPSSSEKLQNINIAHSPLPASETTKPL 450 DAGDD I IPLDFSSGD---SDKP YE DVPL YNDVHLHSTSNKLE--STPVTPLPASEML KPL 458 UKGD IISLDFNNSD--SD IOLL KEVPL KNDVKL PSSSEKLP---LSLSPLTPSDSIP LI 459 TPGD A I ISLDF GNQN-------FEESSAYGKA ILP--------------PSQPWATEL 472

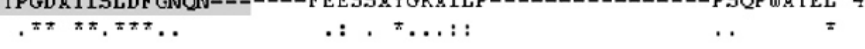

RS-SADPALNQEVALKLE PNPESLELSFTMPQIQDQTPSPSDGSTRQSSPE---------- 512 RS-SADPALNOEVALKLEPNPESLELSF TMPQIQDQP PSPSDGSTRQSSPE--------- 516 RSTS R.DPALNOEVALKL E PNPESLELSF TMPOIODOP ASPSDGSTROSSPE--------- 513

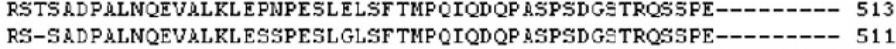
RS-SADPALNQEVALKLESSPESLGLSFTMPQIQDQP ASPSDGSTRQSSPERLLQENVNT 520 RS-N R.D PALNR EVVSKL E PNTE TLELSF TMPQVQEQP TSPSD ASTSQSSPE--------- 510 RS-NVDP QLNR FVVIKNESNPEOLFL \&FTIPOPS-KPGSPSD IGSSOSSTE--------- 507 TK-------_---LETGGEDFPFSS ASDRVPDPTNTPSTSGLGSSGPN-------- 506 RS-----------HSTQSEAGSLPAF TVPQAAAPGSTTPSATSSSSSCST--------- 481 :. $\quad$ : . . : * . . . .

-----PNSPSE YCF YVDSD MVNEF KLELVE KLF AEDTEAKNPFSTQ--DTDLDLEML APY 555 -----PNSPSE YCFYVDSD MVNEF KLELVE KLF AEDTEAKNPFSTQ--DTDLDLEML APY 559 -----PNSPSE YCFDVDSDHVNEF KLELVEKLF AEDTEAKNPFSTQ--DTDLDLEMLAPY 556 ----- PNSPSE YCF DVDSD MVNVF KLELVE KLF AED TE AKNPF SAQ--DTDLDLEML APY 554 PNFSOPNSPSE YCFDUDSDMUNUF KLELUE KLF \&EDTE AKNPFSTO--DTDLDLEML גPY 578 -----PSSPND YCFDVDND HANEF KLELVEKLF A IDTEAKNPFSTQ--ETDLDLEMLAPY 553 -----PSSPPE YCFNVDGE I ASEF KLDLVE KLF AIDTEVKAPF TTQ--ETDLDLEMLAPY 550 ------SPHD YGFPVEPD ISSEF KLDLVEKLF \&IDTEAKTPFSTQP-MEDLDLEML APY 558 -----PNSPED YYTSLD----NDL KIEVIEKLF ÀMD TEAKDQCSTQTDFNELDLETLAPY 532

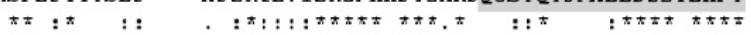

IPND-DDFQLRSFDQLSPLESSSASPESASPQSTVTVFQQTQIQEPTAN----גTTTTAT 620 IPMD-DDFOLRSFDOLSPLESSS ASPES LSSPOSTVTVFOOTOIOEPTAN----DTTTTAT 624 IPUD-DDFOLRSTDOLSPLESSSTSPQSTS---TITVFQPTP UFEPPVT----TTTPAAT 618 IPKD-DDFQLRSFDQLSPLESNSPSPPSVS---TVTGFQQTQLQKPTITVTAATATTATT 620 IPUD-DDFQLRSFDQLSPLESNSPSPPSUS---TVTGFQQTQLQKPTIT---ATKTTTAT 631 IPUD-DDFQL RSFDQLSFLESSSSGSONA----TITILQQTQTF-----------STh 606 IPND-DDFQLRTFDQLSSLECDSSNPQTFQ---SKS ALFQ------------------SIS 599 IPUD-DDFQLRIPSPLDPLPSATHSVSAMS-----SLFQP------------------LPS 595 IP MD GEDFQL SP ICPEE RL LAENPQSTPQHCTSAMTN IFQPL AFVAPHSFFLLDKFQQQL 592

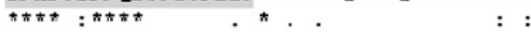

Fig. 2. Multiple sequence alignment of ODD domains in HIF1 $\alpha$ homologs from different species. Multiple sequence alignment was performed using the ClustalW program. The two boxed regions indicate the location of highly conserved NODD and CODD motifs. 
the CODD peptide and p53 DBD were tethered with a force constant of 10 , and the integration time step was $1 \mathrm{fs}$. Finally, the structure was further subjected to conjugate gradient minimization until convergence to 0.01 $\mathrm{kcal} / \mathrm{mol} / \AA$. Molecular dynamics calculations and energy minimization of the complex molecule were carried out with a consistent valence forcefield. Figures were prepared by using the software Insightll.

\section{Results and Discussion}

Two functionally independent sequence motifs, NODD and CODD, in the HIF1 $\alpha$ ODD domain were shown to bind to partner proteins, such as pVHL and p53 (Hansson et al., 2002; Hon et al., 2002). To further investigate the sequence conservation between $\mathrm{HIF} 1 \alpha$ homologs from different species, we performed multiple sequence alignment with HIF1 $\alpha$ homologs from human, monkey, pig, rat, mouse, chicken, frog (Xenopus laevis), and zebrafish (Fig. 2). The sequence alignment by the program ClustalW (Larkin et al., 2007) demonstrates that there are two sequence motifs (NODD and CODD) that display noticeably high conservation of the primary sequence across different species and isoforms. The NODD motif corresponds to residues $387 \sim 420$, whereas the CODD motif encompasses residues $549 \sim 582$. In particular, the leucine-alanine-proline (LAP) motif, which contains the hydroxylation sites $\mathrm{Pro}^{402}$ and $\mathrm{Pro}^{564}$, is common in both the NODD and CODD motifs. On the other hand, only the CODD motif is conserved in human HIF3 $\alpha$ (Hara et al., 2001) (data not shown). The absolutely conserved amino acid residues in the CODD motif include residues 557 560, 562 569, and 571 574

A

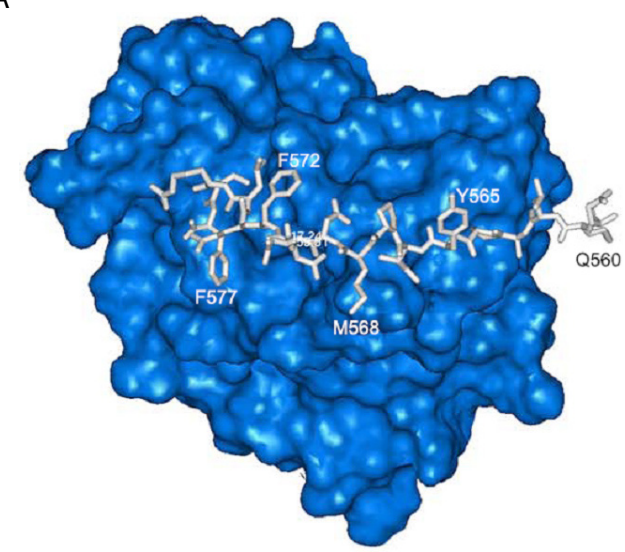

(Fig. 2). It is interesting to see how the same sequence motif is capable of recognizing different partner proteins with high specificity. Also, it remains unclear why the two functionally independent $\mathrm{N}$ - and C-terminal ODD motifs in HIF1 $\alpha$ are necessary for binding to partner proteins such as $\mathrm{pVHL}$ and $\mathrm{p} 53$. One possibility is that the presence of two redundant sequence motifs may be associated with the oligomeric status of the partner proteins (such as a tetramer, in the case of p53) (Hansson et al., 2002; Jeffrey et al., 1995).

The peptide-based binding screening by Hansson et al. identified that the peptides that encompass the CODD motif bind to the p53 DBD with micromolar affinity in solution (Hansson et al., 2002). A previous chemical shift analysis for the CODD peptide by NMR showed that the conformation of the hydroxylated CODD peptide was nearly identical to that of the unmodified CODD peptide in solution (Hon et al., 2002). Therefore, on the basis of the extended structure of the hydroxylated peptide, we generated an initial model of the CODD peptide (residues $560 \sim 577$ ) for docking and molecular dynamics simulation. Based on previous chemical shift perturbation data (Hansson et al., 2002), we built an initial structural model of the CODD-p53 DBD complex and carried out molecular dynamics simulation for the structure of the CODD-p53 DBD complex.

Fig. 3 shows the structure of the CODD-p53 DBD complex that was derived from the molecular dynamics simulation. As shown in Fig. 3A, the CODD peptide molecule occupies the DNA-binding site of the p53 DBD. The structural model for the CODD-p53 DBD complex is in good agreement with previous chemical shift perturbation data. Among the p53 DBD residues that show
B

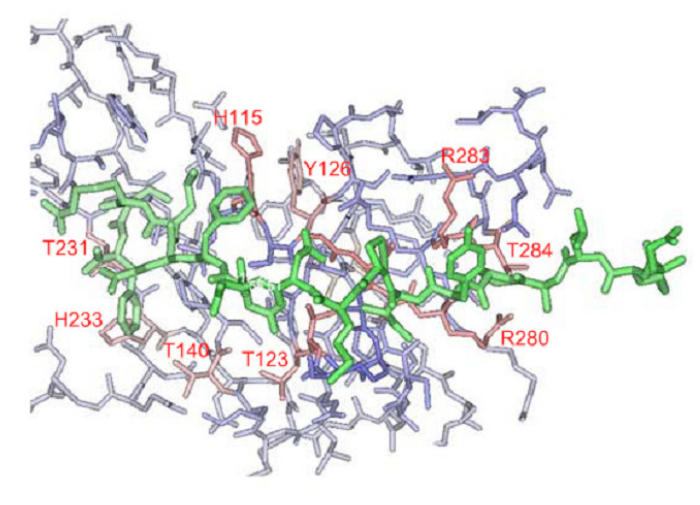

Fig. 3. Structural model for HIF1 $\alpha$ CODD in complex with the p53 DBD. (A) The structure of the CODD peptide (white) bound to the Conolly surface of a p53 DBD molecule (blue). (B) The interaction of the CODD peptide with the residues in the p53 DBD. The p53 DBD residues that showed significant chemical shift perturbations (Hansson et al., 2002) are colored in red. 
significant chemical shift perturbations (Hansson et al., 2002), $\mathrm{His}^{115}, \mathrm{Thr}^{118}, \mathrm{Thr}^{231}, \mathrm{Val}^{122}$, $\mathrm{His}^{233}, \mathrm{Arg}^{280}$, and $\mathrm{Thr}^{284}$ are in direct contact with the CODD peptide (Fig. $3 \mathrm{~B}$, Table 1). On the other hand, the chemical shift perturbations in $\mathrm{Tyr}^{126}$ and $\mathrm{His}^{178}$ are likely to arise not from direct contact but from conformational change, because they are distant from the binding site. The binding of the CODD peptide to the p53 DBD is mediated at two different sites of the p53 DBD. The residues $564 \sim 571$ in the CODD peptide bind to the primary binding site, and residues $572 \sim 577$ bind to the secondary binding site. The primary binding site of the p53 DBD is composed of loop 1 and helix 2 , and the secondary site consists of the $\beta$-strands 3,5 , and 8 . The interactions between the CODD peptide and the p53 DBD are mainly hydrophobic. The high binding specificity of the complex seems to be gained through the extensive involvement of side chains of the CODD peptide that are in direct contact with the p53 DBD, which is similar to what has been observed for the CODD-pVHL complex. In conclusion, the highly conserved CODD motif provides a conserved binding mode and an elaborate binding specificity for the recognition of p53.

Table 1. Major Van der Waals contacts for the HIF1 $\alpha$ CODD-p53 DBD complex

\begin{tabular}{cl}
\hline HIF1 $\alpha$ CODD peptide residues & p53 DBD residues \\
\hline Pro $^{564}$ & $\mathrm{Arg}^{280}, \mathrm{Arg}^{283}, \mathrm{Thr}^{284}$ \\
$\| \mathrm{Ie}^{566}$ & $\mathrm{Thr}^{118}$ \\
$\mathrm{Asp}^{571}$ & $\mathrm{Ser}^{116}, \mathrm{Val}^{122}$ \\
$\mathrm{Phe}^{572}$ & $\mathrm{His}^{115}$ \\
$\mathrm{Leu}^{574}$ & $\mathrm{His}^{115}$ \\
$\mathrm{Phe}^{577}$ & $\mathrm{Thr}^{231}, \mathrm{His}^{233}$ \\
\hline
\end{tabular}

On the basis of the three-dimensional structure model, we investigated the specific functional roles of highly conserved residues in the CODD motif for the recognition of the p53 DBD. The major binding determinants of the CODD motif that are involved in direct contact with the p53 DBD are listed in Table 1. In particular, among the highly conserved residues, the residues $\mathrm{Pro}^{564}$, Ile ${ }^{566}$, $\mathrm{Phe}^{572}$, Leu ${ }^{574}$, and Phe ${ }^{577}$ participate in key hydrophobic interactions and major van der Waals contacts with the p53 DBD residues. On the other hand, Asp $^{571}$ forms a hydrogen bond with $\operatorname{Ser}^{116}$ in the p53 DBD. The other conserved residues seem to play a scaffolding role by precisely positioning the contacting residues for optimal interaction with the p53 DBD. The major binding determinants for $\mathrm{pVHL}$ binding differ slightly from those for p53 binding, indicating that the specific role of each conserved residue, albeit within the same CODD motif, is dependent on the partner protein. This may explain how the same sequence motif is capable of recruiting different partner proteins with high specificity.

One of the most striking differences is that hydroxylation at $\mathrm{Pro}^{564}$ is essential for binding $\mathrm{PVHL}$ but not for binding the p53 DBD. The steady-state affinity constant of VCB ( $\mathrm{pVHL}$, elongins $\mathrm{C}$ and $\mathrm{B}$ ) for the unmodified CODD peptide was estimated to be approximately 1000-fold less than the affinity for the hydroxylated CODD peptide (Hon et al., 2002). This drastic reduction in binding affinity stems from the loss of two hydrogen bonds due to the absence of a hydroxyl group in Pro ${ }^{564}$. Contrary to this, binding of the CODD motif to the p53 DBD does not depend on the hydroxylation of $\mathrm{Pro}^{564}$. Fig. 4 shows a structural comparison of the CODD-p53 DBD complex with the CODD-pVHL complex. $\mathrm{Pro}^{564}$ is rather solvent-exposed on the shallow binding groove of
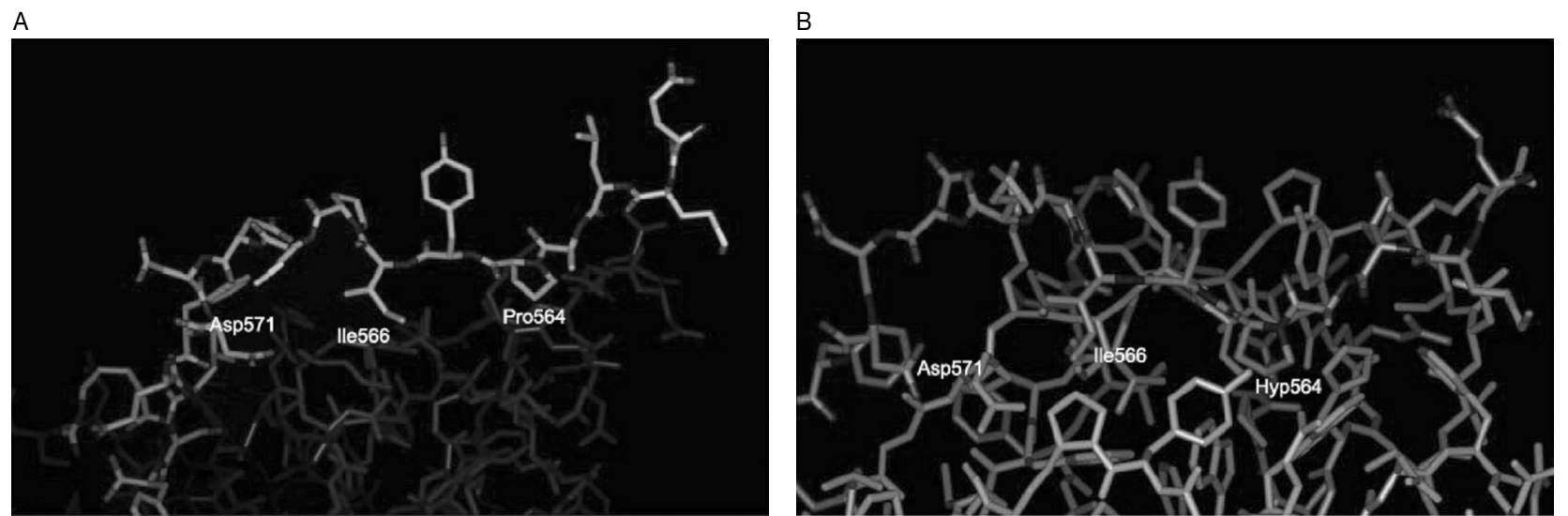

Fig. 4. Structural comparison of the HIF1 $\alpha$ CODD-p53 DBD complex (A) with the HIF1 $\alpha$ CODD-pVHL complex (B). In panel (B), the structure of the hydroxylated peptide bound to pVHL was drawn using the coordinates of PDB code 1LQB (Hon et al., 2002). 
the p53 DBD (Fig. 4A), whereas Hyp ${ }^{564}$ is deeply buried in the deep binding pocket of pVHL (Fig. 4B). This structural difference in the binding pocket may explain why the hydroxylation of $\mathrm{Pro}^{564}$ is required, not for p53 DBD binding, but for pVHL binding.

In conclusion, the multiple sequence alignment of ODD domains of many HIF1 $\alpha$ homologs shows that the NODD and CODD motifs are highly conserved between various species. This evolutionary sequence conservation correlates with the functional importance of the NODD and CODD motifs for their recognition of partner proteins such as $\mathrm{pVHL}$ and $\mathrm{p} 53$. The structure of the HIF1 $\alpha$ CODD motif in complex with the p53 DBD from our molecular dynamics calculation shows that the side chains of highly conserved amino acids are in extensive contact with the residues in the p53 DBD, demonstrating specific functional roles of major binding determinants in the HIF1 $\alpha$ CODD motif. The structure also provides useful information for designing small molecules that modulate HIF1 $\alpha$-p53 interactions in tumor hypoxia.

\section{Acknowledgements}

This work was supported by the Korea Research Foundation Grant funded by the Korean Government (MOEHRD) (KRF-2005-041-E00070), a grant from KRIBB Research Initiative Program, and a grant from the National R\&D Program for Cancer Control, Ministry of Health \& Welfare, Republic of Korea (0720130).

\section{References}

Bruick, R.K., and McKnight, S.L. (2002). Transcription. Oxygen sensing gets a second wind. Science 295, 807-808.

Fels, D.R., and Koumenis, C. (2005). HIF-1 $\alpha$ and p53: the ODD couple? Trends Biochem. Sci. 30, 426-429.

Hansson, L.O., Friedler, A., Freund, S., Rudiger, S., and Fersht, A.R. (2002). Two sequence motifs from HIF-1 $\alpha$ bind to the DNA-binding site of p53. Proc. Natl. Acad. Sci. USA, 99, 10305-10309.

Hara, S., Hamada, J., Kobayashi, C., Kondo, Y., and Imura, N. (2001). Expression and characterization of hypoxia-inducible factor (HIF)-3 $\alpha$ in human kidney: suppression of HIF-mediated gene expression by HIF-3 $\alpha$. Biochem. Biophys. Res. Commun. 287, 808-813.

Hon, W.C., Wilson, M.I., Harlos, K., Claridge, T.D., Schofield, C.J., Pugh, C.W., Maxwell, P.H., Ratcliffe, P.J., Stuart, D.I., and Jones, E.Y. (2002). Structural basis for the recognition of hydroxyproline in $\mathrm{HIF}-1 \alpha$ by $\mathrm{pVHL}$. Nature 417, 975-978.
Ivan, M., Kondo, K., Yang, H., Kim, W., Valiando, J., Ohh, M., Salic, A., Asara, J.M., Lane, W.S., and Kaelin, W.G., Jr. (2001). HIF-1 $\alpha$ targeted for VHL-mediated destruction by proline hydroxylation: implications for $\mathrm{O} 2$ sensing. Science 292, 464-468.

Jaakkola, P., Mole, D.R., Tian, Y.M., Wilson, M.I., Gielbert, J., Gaskell, S.J., Kriegsheim, A., Hebestreit, H.F., Mukherji, M., Schofield, C.J., Maxwell, P.H., Pugh, C.W., and Ratcliffe, P.J. (2001). Targeting of HIF1 $\alpha$ to the von Hippel-Lindau ubiquitylation complex by $\mathrm{O}_{2}$-regulated prolyl hydroxylation. Science 292, 468-472.

Jeffrey, P.D., Gorina, S., and Pavletich, N.P. (1995). Crystal structure of the tetramerization domain of the p53 tumor suppressor at 1.7 angstroms. Science 267, 1498-1502.

Jeong, J.W., Bae, M.K., Ahn, M.Y., Kim, S.H., Sohn, T.K., Bae, M.H., Yoo, M.A., Song, E.J., Lee, K.J., and Kim, K.W. (2002). Regulation and destabilization of HIF-1 $\alpha$ by ARD1-mediated acetylation. Cell 111, 709-720.

Jiang, B.H., Rue, E., Wang, G.L., Roe, R., and Semenza, G.L. (1996). Dimerization, DNA binding, and transactivation properties of hypoxia-inducible factor $1 . \mathrm{J}$. Biol. Chem. 271, 17771-17778.

Lando, D., Peet, D.J., Whelan, D.A., Gorman, J.J., and Whitelaw, M.L. (2002). Asparagine hydroxylation of the HIF transactivation domain a hypoxic switch. Science 295, 858-861.

Larkin, M.A., Blackshields, G., Brown, N.P., Chenna, R., McGettigan, P.A., McWilliam, H., Valentin, F., Wallace, I.M., Wilm, A., Lopez, R., Thompson, J.D., Gibson, T.J., and Higgins, D.G. (2007). Clustal W and Clustal X version 2.0. Bioinformatics 23, 2947-2948.

Lee, J.W., Bae, S.H., Jeong, J.W., Kim, S.H., and Kim, K.W. (2004). Hypoxia-inducible factor (HIF-1) $\alpha$ : its protein stability and biological functions. Exp. Mol. Med. 36, 1-12.

Min, J.H., Yang, H., Ivan, M., Gertler, F., Kaelin, W.G., Jr., and Pavletich, N.P. (2002). Structure of an HIF-1 $\alpha-p V H L$ complex: hydroxyproline recognition in signaling. Science 296, 1886-1889.

Sanchez-Puig, N., Veprintsev, D.B., and Fersht, A.R. (2005). Binding of natively unfolded HIF-1 $\alpha$ ODD domain to p53. Mol. Cell. 17, 11-21.

Schofield, C.J. and Ratcliffe, P.J. (2004). Oxygen sensing by HIF hydroxylases. Nat. Rev. Mol. Cell. Biol. 5, 343354.

Semenza, G.L. (1999). Regulation of mammalian O2 homeostasis by hypoxia-inducible factor 1. Annu. Rev. Cell. Dev. Biol. 15, 551-578.

Semenza, G.L. (2001). HIF-1, O(2), and the 3 PHDs: how animal cells signal hypoxia to the nucleus. Cell. 107, 1-3.

Semenza, G.L. (2003). Targeting HIF-1 for cancer therapy. Nat. Rev. Cancer. 3, 721-732.

Wang, Y., Rosengarth, A., and Luecke, H. (2007). Structure of the human p53 core domain in the absence of DNA. Acta. Crystallogr. D. Biol. Crystallogr. 63, 276-281. 